\title{
Children with Hashimoto's Thyroiditis Have Increased Intestinal Permeability: Results of a Pilot Study
}

\author{
(D) Banu Küçükemre Aydın1, (D) Melek Yıldız1, (D) Abdurrahman Akgün1, (D) Neval Topal1, (D) Erdal Adal2, (D) Hasan Önal1 \\ 1 University of Health Sciences Turkey, Kanuni Sultan Süleyman Training and Research Hospital, Unit of Pediatric Endocrinology and Metabolism, \\ istanbul, Turkey \\ 2Medipol University Faculty of Medicine, Department of Pediatric Endocrinology and Metabolism, Istanbul, Turkey
}

\begin{abstract}
What is already known on this topic?
Increased intestinal permeability (IIP) was shown to precede the onset of several autoimmune disorders. Although Hashimoto's thyroiditis (HT) is the most common autoimmune disorder, the role of IIP in its pathogenesis had received little attention. Exact disease mechanisms are poorly elucidated in HT.
\end{abstract}

\section{What this study adds?}

Children and adolescents with HT had increased zonulin levels compared to their age, gender and body mass index matched peers with congenital hypothyroidism. Higher zonulin levels suggested IIP in these patients.

\begin{abstract}
Increased intestinal permeability (IIP) precedes several autoimmune disorders. Although Hashimoto's thyroiditis (HT) is the most common autoimmune disorder, the role of IIP in its pathogenesis had received little attention. Zonulin plays a critical role in IIP by modulating intracellular tight junctions. Rise of serum zonulin levels were shown to indicate IIP in human subjects. In this case-control study, we examined the hypothesis that patients with HT have IIP. We studied 30 children and adolescents with HT, and 30 patients with congenital hypothyroidism (CH) matched for age, gender and body mass index (BMI). Serum zonulin levels, free thyroxine (fT4), thyroid stimulating hormone (TSH), anti-thyroglobulin antibody and anti-thyroid peroxidase antibody were measured. Zonulin levels were significantly higher in patients with HT than patients with $\mathrm{CH}(59.1 \pm 22.9 \mathrm{ng} / \mathrm{mL} v \mathrm{ss} .43 .3 \pm 32.9 \mathrm{ng} / \mathrm{mL}, \mathrm{p}=0.035)$. In patients with $\mathrm{HT}$, zonulin levels were positively correlated with weight $(r=0.406, p=0.03)$, BMI $(r=0.486, p=0.006)$ and levothyroxine dose $(r=0.463$, $\mathrm{p}=0.02)$. In patients with $\mathrm{CH}$, zonulin levels were positively correlated with age $(r=0.475, p=0.008)$, weight $(r=0.707, p<0.001)$, BMI $(r=0.872, p<0.001)$ and levothyroxine dose $(r=0.485, p=0.007)$. After adjusting for age, weight, TSH and fT4 levels, serum zonulin was only associated with levothyroxine dose in patients with $\mathrm{HT}\left(\mathrm{R}^{2}=0.36, \mathrm{p}=0.05\right)$. In patients with $\mathrm{CH}$, only weight was associated with zonulin levels $\left(R^{2}=0.62, p<0.001\right)$. In conclusion, higher zonulin levels in children and adolescents with HT suggested IIP in these patients. Additionally, the association between zonulin levels and levothyroxine dose might imply a relationship between serum zonulin and disease severity.
\end{abstract}

Keywords: Autoimmune thyroiditis, congenital hypothyroidism, zonulin, increased intestinal permeability

Address for Correspondence: Banu Küçükemre Aydın MD, University of Health Sciences Turkey, Kanuni Sultan Süleyman Training and Research Hospital, Unit of Pediatric Endocrinology and Metabolism, İstanbul, Turkey Phone: + 902124041500 E-mail: bkucukemre@yahoo.com ORCID: orcid.org/0000-0003-0859-1565

This study was presented in $58^{\text {th }}$ European Society of Paediatric Endocrinology meeting and the abstract was published in Hormone Research in Paediatrics, 2019;91(Suppl 1):270.

${ }^{\circ}$ Copyright 2020 by Turkish Pediatric Endocrinology and Diabetes Society

The Journal of Clinical Research in Pediatric Endocrinology published by Galenos Publishing House.
Conflict of interest: None declared Received: 15.11 .2019 Accepted: 23.01.2020 


\section{Introduction}

Both genetic predisposition and environmental factors play some role in triggering Hashimoto thyroiditis (HT), but the exact mechanisms are still not fully understood. Increased intestinal permeability (IIP) was shown to be a constant and early feature of several autoimmune disorders (1). Although HT is the most common autoimmune disorder worldwide, the role of IIP in its pathogenesis had received little attention.

Human zonulin regulates intestinal permeability by disassembling the tight junctions (TJ) (2). Zonulin was shown to play a key role in IIP, when up regulated (3). Increased zonulin levels were reported to be correlated with IIP in vivo and changes in claudin-1, claudin-2, and myosin IXB gene expression (4). Higher zonulin expression was reported in the intestinal tissues of patients with many autoimmune disorders (4). Increased serum zonulin levels were detected in human subjects during the pre-diabetic stage and preceded the onset of type 1 diabetes (4). In a rat model, zonulin-dependent IIP was shown to precede the onset of type 1 diabetes by 2-3 weeks (3). Moreover, oral administration of the zonulin inhibitor (AT-1001) to these rats blocked autoantibody formation, zonulin-mediated IIP, and finally reduced the incidence of diabetes (3). AT1001 competitively blocks the apical zonulin receptor and prevents the opening of the TJ (2).

In this study, the hypothesis that patients with HT have IIP was investigated. To this end, blood zonulin levels were examined in patients with HT and patients with congenital hypothyroidism $(\mathrm{CH})$ were used as a control group. To the best of our knowledge this is the first study which examines zonulin levels in patients with HT.

\section{Methods}

This was a case-control study in a group of 30 consecutive children and adolescents with HT, and 30 age-, genderand BMI-matched patients with $\mathrm{CH}$. The diagnosis of HT was based on positive anti-thyroid peroxidase antibody (TPO), anti-TG (antibodies against thyroglobulin) levels and the presence of a typical thyroiditis ultrasound pattern. Obese patients and patients with acute or other chronic diseases were excluded from the study. Informed consent was obtained in accordance with a protocol approved by the Institutional Review Board of Sadi Konuk Training and Research Hospital (2017-207).

\section{Anthropometric Measurements}

Heights were measured in the standing position with bare feet, using Harpenden equipment (Holtain, United
Kingdom), and an electronic scale sensitive to 0.1 kilogram (Seca, Germany) was used for weight measurements. Body mass index (BMI) was calculated according to the standard formula. Height, weight and BMI z-scores standard deviation score (SDS) were calculated according to national standards (5). Obesity was defined as a BMI-SDS $\geq 2$ SDS.

\section{Laboratory Evaluation}

Venous blood samples were collected for zonulin, free thyroxine (fT4), thyroid stimulating hormone (TSH), anti-TPO, and anti-TG levels. Samples were immediately processed and sera were stored at $-80^{\circ} \mathrm{C}$ for subsequent batch analysis. Zonulin levels were measured by human zonulin enzymelinked immunosorbent assay kit (ELISA, Elabscience, Houston, Texas, USA). Levels of fT4, TSH, anti-TPO and anti-TG were measured by electrochemiluminescence immunoassay (ECLIA, Cobas e602, Roche Diagnostics, Basel, Switzerland).

Thyroid ultrasound results were retrieved from the patient's files.

\section{Statistical Analysis}

The Shapiro-Wilk test was used to determine whether variables were normally distributed. Data were presented as mean \pm SDS. Comparisons were made by using independent samples t-test or $\chi^{2}$ test. Pearson analysis was used to determine correlations between zonulin levels and other clinical parameters. Separate linear regression models were created to examine the associations of blood zonulin levels in patients with HT and $\mathrm{CH}$. All statistical analyses were conducted with Statistical Package for the Social Sciences version 15.0 (IBM Inc., Chicago, IL., USA). Statistical significance was defined as $\mathrm{p} \leq 0.05$.

\section{Results}

\section{Patients' Characteristics}

Serum zonulin levels were significantly higher in patients with HT than patients with $\mathrm{CH}$ (Table 1, Figure 1). Age, gender, weight SDS, height SDS and BMI-SDS were not different between the groups (Table 1). Serum fT 4 levels and daily levothyroxine dose were higher in patients with $\mathrm{CH}$, but TSH levels were not different between the groups (Table 1). Anti-TPO and anti-TG levels were higher in patients with HT (Table 1). Thyroid ultrasonography in patients with HT revealed heterogeneous parenchyma in 17 patients and combined heterogeneous and pseudo-nodular parenchyma in 13 (Table 1). In patients with $\mathrm{CH}$, ultrasonography showed agenesis of the thyroid gland in 15 patients, heterogeneous 
Table 1. Comparison of the clinical and laboratory parameters of the patients with Hashimoto thyroiditis and with congenital hypothyroidism (all values are means \pm standard deviations, except if otherwise stated)

\begin{tabular}{|c|c|c|c|}
\hline & $\begin{array}{l}\text { Hashimoto } \\
\text { thyroiditis } \\
\mathrm{n}=30\end{array}$ & $\begin{array}{l}\text { Congenital } \\
\text { hypothyroidism } \\
\mathrm{n}=30\end{array}$ & $\mathrm{p}$ value \\
\hline Age (years) & $12.6 \pm 2.7$ & $11.3 \pm 3.4$ & 0.11 \\
\hline Gender (F/M) & $25 / 5$ & $22 / 8$ & 0.35 \\
\hline $\begin{array}{l}\text { L-thyroxine dose } \\
(\mu g / \mathrm{kg} / \text { day })\end{array}$ & $1.2 \pm 0.84$ & $1.9 \pm 0.59$ & $<0.001$ \\
\hline Weight SDS & $-0.32 \pm 1.3$ & $0.01 \pm 1.1$ & 0.30 \\
\hline Height SDS & $-0.40 \pm 1.1$ & $-0.20 \pm 1.1$ & 0.42 \\
\hline BMI-SDS & $-0.11 \pm 1.2$ & $0.09 \pm 1.3$ & 0.54 \\
\hline fT4 (ng/dL) & $1.28 \pm 0.2$ & $1.45 \pm 0.2$ & 0.004 \\
\hline TSH (mIU/L) & $6.5 \pm 5.9$ & $7.7 \pm 6.3$ & 0.37 \\
\hline Anti-TPO (IU/mL) & $268.2 \pm 201$ & $11.4 \pm 3$ & $<0.001$ \\
\hline Anti-TG (IU/mL) & $641.4 \pm 1076$ & $12.3 \pm 3.1$ & $<0.001$ \\
\hline \multicolumn{4}{|l|}{ USG } \\
\hline Normal & 0 & 12 & \\
\hline Agenesis & 0 & 15 & \\
\hline Heterogeneous & 17 & 3 & $<0.001$ \\
\hline $\begin{array}{l}\text { Heterogeneous and } \\
\text { Pseudo-nodular }\end{array}$ & 13 & 0 & \\
\hline Zonulin (ng/mL) & $59.1 \pm 22.9$ & $43.3 \pm 32.9$ & 0.035 \\
\hline
\end{tabular}

Anti-TG: anti-thyroglobulin antibody, Anti-TPO: anti-thyroid peroxidase antibody, BMI: body mass index, F: female, fT4: free thyroxine, L-thyroxine: levothyroxine, M: male, SDS: standard deviation score, TSH: thyroid stimulating hormone, USG: ultrasonography

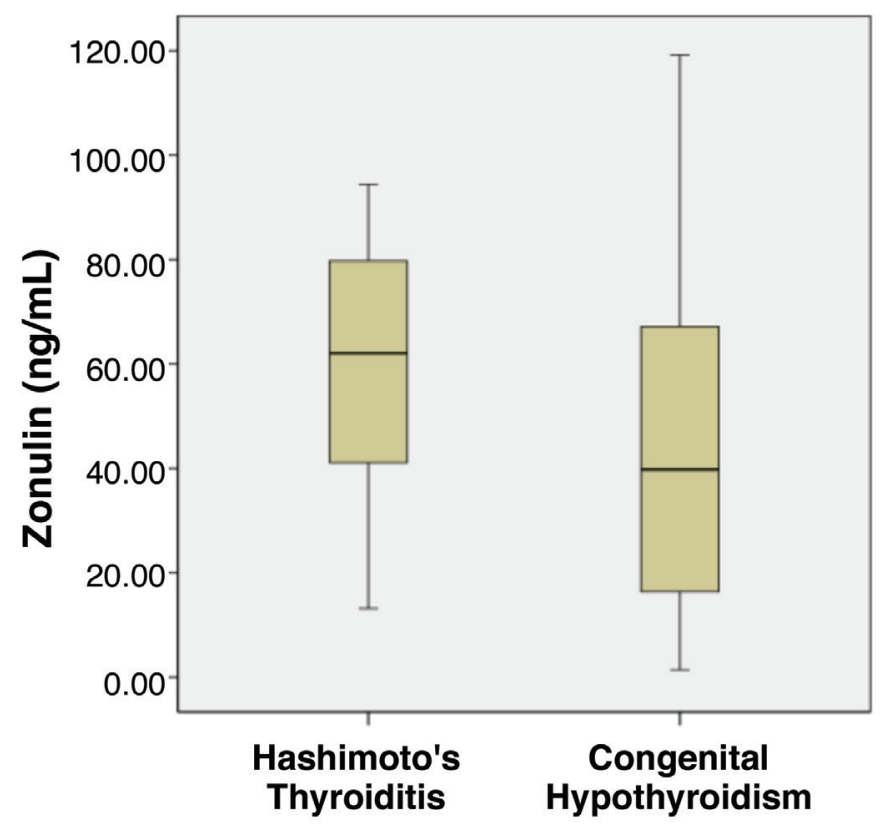

Figure 1. Serum zonulin levels (ng/mL) in patients with Hashimoto thyroiditis and congenital hypothyroidism parenchyma in three and normal thyroid gland in 12 patients (Table 1).

\section{Correlation Analyses}

In patients with $\mathrm{HT}$, zonulin levels were positively correlated with weight $(r=0.406, p=0.03)$, weight SDS $(r=0.377$, $p=0.04)$, BMI $(r=0.486, p=0.006)$, BMI-SDS $(r=0.419$, $p=0.02)$ and levothyroxine dose $(r=0.463, p=0.02)$. There was no significant correlation between zonulin levels and anti-TPO or anti-TG levels $(r=-0.174, p=0.4$ and $r=0.295$, $\mathrm{p}=0.1$, respectively).

In patients with $\mathrm{CH}$, there were strong positive correlations between zonulin levels and age $(r=0.475, p=0.008)$, weight $(r=0.707, p<0.001)$, weight SDS $(r=0.532, p=0.002)$, BMI $(r=0.872, p<0.001)$, BMI-SDS $(r=0.681, p<0.001)$ and levothyroxine dose $(r=0.485, p=0.007)$.

\section{Regression Analyses}

In patients with HT, serum zonulin was only associated with levothyroxine dose after adjusting for age, weight, TSH and fT4 levels, but with a borderline $p$-value $\left(R^{2}=0.36, p=0.05\right)$.

When the patients with $\mathrm{CH}$ were put into the same regression model, there was no significant association between zonulin level and levothyroxine dose $(p=0.4)$. However, serum zonulin was strongly associated only with weight in these patients $\left(R^{2}=0.62, p<0.001\right)$.

\section{Discussion}

Intestinal mucosa is the largest contact site for the host's immune system and exterior antigens, such as food antigens, bacteria, pathogens, and toxins (6). Increase in permeability can cause an exposure of sub-mucosal immune cells to these various antigens and may lead to the development of autoimmune disorders $(6,7)$. Indeed, previous studies have shown that the increased permeability is a key pathogenic component rather than an epiphenomenon of autoimmune disorders $(3,4)$. It was hypothesized that intestinal permeability is also increased in HT (8), but to the best of our knowledge, to date, no study has addressed this issue in these patients. Only in a letter to the editor, Sasso et al (9) described ultrastructural morphological changes of distal duodenum enterocytes in four patients with HT. They also reported IIP, evaluated by a lactulose/mannitol test, in these four patients (9). In our study, it has been demonstrated that children and adolescents with HT had increased zonulin levels compared to their age, gender and BMI matched peers with $\mathrm{CH}$.

Intestinal TJ create gradients for the optimal absorption of nutrients and control tolerance/immunity balance to 
non-self antigens (10). Zonulin, a physiological modulator of $\mathrm{TJ}$, is involved in trafficking of macromolecules and, therefore, in balance between tolerance and immune response $(2,11)$. Sturgeon et al (12) identified zonulin as a master regulator of intercellular TJ. They showed that, there was IIP present in zonulin transgenic mice compared to wild-type mice, which was associated with upregulation of zonulin gene expression. Moreover, treatment with AT1001 (Larazotide acetate) reduced intestinal permeability both in vivo and ex vivo and reverted morbidity and mortality in these zonulin transgenic mice. Their data demonstrated that zonulin-dependent small intestinal barrier dysfunction is an early step leading to the break of tolerance with subsequent development of colitis (12). Similarly, Arrieta et al (13) reported that IL10 gene-deficient mice treated with AT-1001, showed a marked decrease in small intestinal permeability and a clear reduction of colitis severity. In addition, several clinical trials reported a potential beneficial effect of AT1001 in patients with celiac disease (14). In a study of 339 type 1 diabetic patients and their first degree relatives, Sapone et al (4) showed that patients with type 1 diabetes and their relatives have elevated serum zonulin levels that correlate with IIP associated with altered genetic expression of intestinal TJ proteins. Zonulin upregulation was shown to precede the onset of diabetes, providing a possible link between IIP, environmental exposure to nonself antigens, and the development of autoimmunity in genetically susceptible individuals for type 1 diabetes (4). Our finding of increased zonulin levels in patients with HT may trigger future studies that may lead to potential new therapies for HT.

Increased zonulin levels have been reported with aging and obesity (15). It has been suggested that IIP may play a critical role in the development of age-related and obesityrelated inflammation and comorbidities. Similarly, we showed that, serum zonulin levels were strongly correlated with age, weight and BMI, in our study group.

Serum zonulin levels were also positively correlated with levothyroxine dose both in patients with $\mathrm{CH}$ and HT. Moreover, in patients with HT, zonulin level was only associated with levothyroxine dose after adjusting for age, weight, TSH and fT4 levels. By contrast, there was no such association in $\mathrm{CH}$ patients. This finding might imply a relationship between serum zonulin levels and disease severity in HT, because in patients with $\mathrm{CH}$, levothyroxine dose is mainly based on the amount of residual thyroid tissue/function, age, body weight, and thyroid hormone levels (16). However, in patients in HT, there is another associate of the levothyroxine dose; degree of the thyroid damage by autoimmune mechanisms (17).
We did not find a significant correlation between anti-TPO or anti-TG antibodies and serum zonulin levels. However, the contribution of anti-TPO antibodies to thyroid damage compared to T-cell and cytokine-mediated apoptosis in HT is minor and anti-TG antibodies do not cause thyroid cell destruction $(18,19)$. Thyroid damage in HT is mostly due to an apoptotic processes combined with CD8 + cell mediated cytotoxicity, changes in cell junctions, and complement activation $(18,19)$.

\section{Study Limitations}

This was a pilot study with small numbers of children. In addition, we did not have a healthy control group to compare zonulin levels in children and adolescents who had normal thyroid function and therefore would not be on levothyroxine therapy.

\section{Conclusion}

Higher zonulin levels in children and adolescents with HT suggested IIP in these patients. Further research is needed to expand these results in larger cohorts to gain more insight into the pathogenesis of HT and to lead to potential new treatments.

\section{Ethics}

Ethics Committee Approval: Institutional Review Board of Sadi Konuk Training and Research Hospital (2017-207).

Informed Consent: Informed consent was obtained in accordance with the protocol approved by the ethics committee.

Peer-review: Externally peer-reviewed.

\section{Authorship Contributions}

Surgical and Medical Practices: Banu Küçükemre Aydın, Melek Yıldız, Abdurrahman Akgün, Neval Topal. Concept: Banu Küçükemre Aydın, Erdal Adal, Hasan Önal, Design: Banu Küçükemre Aydın, Erdal Adal, Hasan Önal, Data Collection or Processing: Banu Küçükemre Aydın, Melek Yıldız, Abdurrahman Akgün, Neval Topal. Analysis or Interpretation: Banu Küçükemre Aydın, Melek Yıldız, Literature Search: Banu Küçükemre Aydın, Melek Yıldız, Abdurrahman Akgün, Neval Topal, Erdal Adal, Hasan Önal, Writing: Banu Küçükemre Aydın, Melek Yıldız, Abdurrahman Akgün, Neval Topal, Erdal Adal, Hasan Önal.

Financial Disclosure: This study was funded by the Scientific Research Projects Coordination Unit of Health Sciences University of Turkey [grant number: 2017/022 (to $\mathrm{HO})]$. 


\section{References}

1. Arrieta MC, Bistritz L, Meddings JB. Alterations in intestinal permeability. Gut 2006;55:1512-1520.

2. Wang W, Uzzau S, Goldblum SE, Fasano A. Human zonulin, a potential modulator of intestinal tight junctions. J Cell Sci 2000;113:44354440 .

3. Watts T, Berti I, Sapone A, Gerarduzzi T, Not T, Zielke R, Fasano A. Role of the intestinal tight junction modulator zonulin in the pathogenesis of type I diabetes in BB diabetic-prone rats. Proc Natl Acad Sci USA 2005;102:2916-2921. Epub 2005 Feb 14

4. Sapone A, de Magistris L, Pietzak M, Clemente MG, Tripathi A, Cucca F, Lampis R, Kryszak D, Carteni M, Generoso M, Iafusco D, Prisco F, Laghi F, Riegler G, Carratu R, Counts D, Fasano A. Zonulin upregulation is associated with increased gut permeability in subjects with type 1 diabetes and their relatives. Diabetes 2006;55:1443-1449.

5. Neyzi O, Bundak R, Gökçay G, Günöz H, Furman A, Darendeliler F, Baş F. Reference Values for Weight, Height, Head Circumference, and Body Mass Index in Turkish Children. J Clin Res Pediatr Endocrinol 2015;7:280-293

6. Virili C, Centanni M. Does microbiota composition affect thyroid homeostasis? Endocrine 2015;49:583-587. Epub 2014 Dec 17

7. Macdonald TT, Monteleone G. Immunity, inflammation, and allergy in the gut. Science 2005;307:1920-1925.

8. Virili C, Fallahi P, Antonelli A, Benvenga S, Centanni M. Gut microbiota and Hashimoto's thyroiditis. Endocr Metab Disord 2018;19:293-300.

9. Sasso FC, Carbonara O, Torella R, Mezzogiorno A, Esposito V, Demagistris L, Secondulfo M, Carratu' R, Iafusco D, Cartenì M. Ultrastructural changes in enterocytes in subjects with Hashimoto's thyroiditis. Gut 2004;53:1878-1880.
10. Fasano A. Zonulin, regulation of tight junctions, and autoimmune diseases. Ann N Y Acad Sci 2012;1258:25-33.

11. Fasano A. Zonulin and its regulation of intestinal barrier function: the biological door to inflammation, autoimmunity, and cancer. Physiol Rev 2011;91:151-175.

12. Sturgeon C, Lan J, Fasano A. Zonulin transgenic mice show altered gut permeability and increased morbidity/mortality in the DSS colitis model. Ann N Y Acad Sci 2017;1397:130-142.

13. Arrieta MC, Madsen K, Doyle J, Meddings J. Reducing small intestinal permeability attenuates colitis in the IL10 gene-deficient mouse. Gut 2009;58:41-48. Epub 2008 Oct 1

14. Khaleghi S, Ju JM, Lamba A, Murray JA. The potential utility of tight junction regulation in celiac disease: focus on larazotide acetate. Therap Adv Gastroenterol 2016;9:37-49.

15. Küme T, Acar S, Tuhan H, Çatlı G, Anık A, Gürsoy Çalan Ö, Böber E, Abac1 A. The Relationship between Serum Zonulin Level and Clinical and Laboratory Parameters of Childhood Obesity. J Clin Res Pediatr Endocrinol 2017;9:31-38. Epub 2016/12/23

16. Duntas LH, Jonklaas J. Levothyroxine Dose Adjustment to Optimise Therapy Throughout a Patient's Lifetime. Adv Ther 2019;36(Suppl 2):30-46. Epub 2019 Sep 4

17. Singh R. Does One Size Fit Everyone? Replacement Dose of Levothyroxine in Long-standing Primary Hypothyroidism in Adults. Indian J Endocrinol Metab 2017;21:404-409.

18. Zaletel K, Gaberšček S. Hashimoto's Thyroiditis: From Genes to the Disease. Curr Genomics 2011;12:576-588.

19. Fröhlich E, Wahl R. Thyroid Autoimmunity: Role of Anti-thyroid Antibodies in Thyroid and Extra-Thyroidal Diseases. Front Immunol $2017 ; 9 ; 8: 521$ 